\title{
Mammogram Analysis Using Two-Dimensional Autoregressive Models: Sufficient or Not?
}

\author{
Sarah Lee ${ }^{1,2, \star}$ and Tania Stathaki ${ }^{3}$ \\ 1 Department of Diabetes, Endocrinology \& Internal Medicine, \\ Guy's, King's and St Thomas' School of Medicine, King's College London, \\ Denmark Hill Campus, New Medical School Building, \\ Bessemer Rd, London. SE5 9PJ UK \\ 2 Brain Image Analysis Unit, Centre for Neuroimaging Sciences, \\ Institute of Psychiatry, De Crespigny Park, London, SE5 8AF, UK \\ 3 Communications and Signal Processing Group, \\ Department of Electrical and Electronic Engineering, \\ Imperial College London, Exhibition Rd, London. SW7 2AZ UK \\ sarah.lee@iop.kcl.ac.uk, tania@imperial.ac.uk
}

\begin{abstract}
Two-dimensional $(2-D)$ autoregressive (AR) models have been used as one of the methods to characterise the textures of tumours in mammograms. Previously, the $2-D$ AR model coefficients were estimated for the block containing the tumour and the blocks in its $3 \times 3$ neighbourhood. In this paper, the possibility of having the estimated set of AR model coefficients of the block containing the tumour as a unique set of AR model coefficients for the entire mammogram is looked into. Based on the information given from the MiniMammography database, the possible number of blocks of the same size of the block containing the tumour is obtained from the entire mammogram and for each block a set of AR model coefficients is estimated using a method that combines both the Yule-Walker system of equations and the Yule-Walker system of equations in the third-order statistical domain. These sets of AR model coefficients are then compared. The simulation results show that $98.6 \%$ of the time we can not find another set of AR model coefficients representing the blocks of pixels in the possible neighbourhood of the entire mammogram for the data (95 mammograms with 5 of them having two tumours) available in the MiniMammography database.
\end{abstract}

\section{Introduction}

The two-dimensional $(2-D)$ autoregressive modelling technique has been used as one of the statistical methods to characterise and analyse the textures in images [4] 2]. The images is divide into blocks of pixels and for each block a set of AR model coefficients is estimated. Alternatively, for the area of interest a set of AR model coefficients is estimated.

\footnotetext{
* The author would like to thank Professors A.G. Constantinides and C. Xydeas for their suggestions and comments. This work was carried out while the author was with Communications and Signal Processing Group, Imperial College London.
} 
A number of methods are available in the literature for estimating the AR model coefficients, including the Yule-Walker system of equations (YW) [1], the Yule-Walker system of equations in the third-order statistical domain (YWT) [8], the combined method using the two aforementioned methods [4], the constrained optimisation formulation with equality constraints (ConEq) 4 and the constrained optimisation formulation with inequality constraints (ConIneq) 44. From [4], the comparison among these methods can be found for synthetic images which are built using a known stable $2-D$ AR model and using a non-Gaussian driving input. The images are also contaminated with an external Gaussian noise. The YW method is capable of estimating the AR model coefficients when the external noise is small. The YWT method can give good estimations when the external noise is large, however, the variances calculated using the estimations from a number of realisations are higher than the YW method. The rest of aforementioned methods were proposed to improve these problems.

In 2] and [4, the $2-D$ AR modelling technique is applied to mammogram analysis. The block containing the tumour was analysed and the coefficients representing the block were claimed to be symmetric. However, by taking a block of the same size of pixels in any of them in its possible neighbourhood, can we find another set of AR model coefficients which have values close to the one representing the tumour block? This is the question we try to answer in this paper.

All mammograms containing a tumour or tumours are taken from the database [5]. There are 95 of them to analyse, including 5 of mammograms containing two tumours. The combined method that employs both the Yule-Walker system of equations and the Yule-Walker system of equations in the third-order statistical domain [4] is used to estimate the AR model coefficients. The reason for choosing this method is that in [4] it was proven that this method is capable of estimating AR model coefficients in both low and high SNR environments.

The paper is organised as follows. In Section 2 , the $2-D$ AR model is revisited. In Sections 3, 4 and 5, the conventional Yule-Walker system of equations, the Yule-Walker system of equations in the third-order statistical domain and the combined method can be found respectively. How the possible neighbourhood around the tumour is defined is explained in Section 6. In Section 7, simulation results can be found. The conclusion and summary are given in Section 8 .

\section{Two-Dimensional Autoregressive Model}

Let us consider a digitised image $x$ of size $M \times N$. Each pixel of $x$ is characterised by its location $[m, n]$ and can be represented as $x[m, n]$, where $1 \leq m \leq M$, $1 \leq n \leq N$ and $x[m, n]$ is a positive intensity (gray level) associated with it. A two-dimensional $(2-D)$ autoregressive (AR) model is defined as [1]

$$
x[m, n]=-\sum_{i=0}^{p_{1}} \sum_{j=0}^{p_{2}} a[i, j] x[m-i, n-j]+w[m, n],
$$

where $[i, j] \neq[0,0], a[i, j]$ is the AR model coefficient, $w[m, n]$ is the input driving noise, and $p_{1} \times p_{2}$ is the order of the model. 
The driving noise, $w[m, n]$, is assumed to be zero-mean, i.e., $E\{w[m, n]\}=0$ and non-Gaussian. The AR model coefficient $a[0,0]$ is assumed to be 1 for scaling purposes, therefore we have $\left[\left(p_{1}+1\right)\left(p_{2}+1\right)-1\right]$ unknown coefficients to solve.

An external zero-mean Gaussian noise, $v[m, n]$, is added onto the system, so that the method will be able to deal with real images which are contaminated with such noise. Mathematically the new system can be written as

$$
y[m, n]=x[m, n]+v[m, n] .
$$

The signal-to-noise ratio (SNR) of the system is calculated by

$$
S N R=10 \log _{10} \frac{\sigma_{x}^{2}}{\sigma_{v}^{2}} \quad d B
$$

where $\sigma_{x}^{2}$ is the variance of the signal and $\sigma_{v}^{2}$ is the variance of the noise.

\section{Yule-Walker System of Equations}

The conventional Yule-Walker equations are given by [1] 6 ]

$$
\sum_{i=0}^{p_{1}} \sum_{j=0}^{p_{2}} a[i, j] r_{y y}[i-k, j-l]=-r_{y y}[-k,-l]
$$

for $k=0, \cdots, p_{1}$ and $l=0, \cdots, p_{2}$, where $[k, l] \neq[0,0],[i, j] \neq[0,0], a[i, j]$ is the AR model coefficient, $1 \leq m \leq M, 1 \leq n \leq N, M \times N$ is the size of the given image and $r_{y y}[i, j]=E\{y[m, n] y[m+i, n+j]\}$.

Equation (4) can be written in vector-matrix form as

$$
\underline{R a}=-\underline{r}
$$

where $\underline{\boldsymbol{R}}$ is a $\left(p_{1} p_{2}+p_{1}+p_{2}\right) \times\left(p_{1} p_{2}+p_{1}+p_{2}\right)$ matrix and $\underline{\boldsymbol{a}}$ and $\underline{\boldsymbol{r}}$ are both $\left(p_{1} p_{2}+p_{1}+p_{2}\right) \times 1$ vectors.

These equations give good AR model coefficient estimations when the SNR is high. However, the error increases with $\sigma_{v}^{2}$.

\section{Yule-Walker System of Equations in the Third-Order Statistical Domain}

The equations that relate the AR model parameters to the third-order moment samples are 6] 8]:

$$
\sum_{i=0}^{p_{1}} \sum_{j=0}^{p_{2}} a[i, j] C_{3 y}([i-k, j-l],[i-k, j-l])=-C_{3 y}([-k,-l],[-k,-l])
$$

for $k=0, \cdots, p_{1}, l=0, \cdots, p_{2}$ and $[k, l] \neq[0,0]$, where $C_{3 y}\left(\left[i_{1}, j_{1}\right],\left[i_{2}, j_{2}\right]\right)=E\left\{y[m, n] y\left[m+i_{1}, n+j_{1}\right] y\left[m+i_{2}, n+j_{2}\right]\right\}$ for zero-mean process $y[m, n]$. 
These equations are insensitive to external Gaussian noise. The equations can be written in matrix form as

$$
\underline{C a}=-\underline{c}
$$

where $\underline{\boldsymbol{C}}$ is a $\left(p_{1} p_{2}+p_{1}+p_{2}\right) \times\left(p_{1} p_{2}+p_{1}+p_{2}\right)$ matrix and $\underline{\boldsymbol{a}}$ and $\underline{\boldsymbol{c}}$ are both $\left(p_{1} p_{2}+p_{1}+p_{2}\right) \times 1$ vectors.

\section{The Method Combining the YW and the YWT}

The method using both the YW and the YWT techniques was defined as [3] [4]

$$
\underline{D}\left(\begin{array}{l}
\underline{R} \\
\underline{C}
\end{array}\right) \underline{a}=-\underline{D}\left(\begin{array}{l}
\underline{r} \\
\underline{c}
\end{array}\right)
$$

where the matrix $\underline{\boldsymbol{R}}$ and vector $\underline{\boldsymbol{r}}$ are defined in (5),

the matrix $\underline{C}$ and vector $\underline{c}$ are defined in (7),

$\underline{D}$ is a diagonal weighting matrix, and

$\underline{\boldsymbol{a}}$ is the vector of the unknown AR model coefficients, $\left[a[0,1], \cdots, a\left[0, p_{2}\right], \cdots, a\left[p_{1}, p_{2}\right]\right]^{T}$.

Let us consider the formulation where the system is contaminated with external Gaussian noise.

$$
\underline{\boldsymbol{R}}_{y y} \underline{\boldsymbol{a}}+\underline{\boldsymbol{r}}_{y y}=\sigma_{v}^{2} \underline{\boldsymbol{I} \boldsymbol{a}}
$$

where the AR model coefficients estimation $\underline{\boldsymbol{a}}$ is obtained from (7) using

$$
\underline{a}=-\underline{C}^{-1} \underline{c} .
$$

Let $\underline{\boldsymbol{r}}_{1}=\underline{\boldsymbol{R}}_{y y} \underline{\boldsymbol{a}}+\underline{\boldsymbol{r}}_{y y}$, where $\underline{\boldsymbol{a}}$ is obtained from (10). The variance of the noise $v[m, n]$ can be calculate using

$$
\sigma_{v}^{2}=\left(\underline{\boldsymbol{a}}^{T} \underline{\boldsymbol{a}}\right)^{-1} \underline{\boldsymbol{a}}^{T} \underline{\boldsymbol{r}}_{1}
$$

The weighting diagonal matrix, $\underline{\boldsymbol{D}}$, is determined as (12).

$$
D[i, i]= \begin{cases}1 & \text { for } 1 \leq i \leq\left(p_{1}+1\right)\left(p_{2}+1\right)-1 \\ \left\lceil 50 \sigma_{v}^{2}\right\rceil \text { for }\left(p_{1}+1\right)\left(p_{2}+1\right) \leq i \leq 2\left(p_{1}+1\right)\left(p_{2}+1\right)-2\end{cases}
$$

where $\lceil x\rceil$ denotes rounding toward infinity.

\section{Possible Neighbourhood Around Tumour}

The main purpose of this paper is to find out whether the set of AR model coefficients that represents the texture of the block containing an tumour can be 


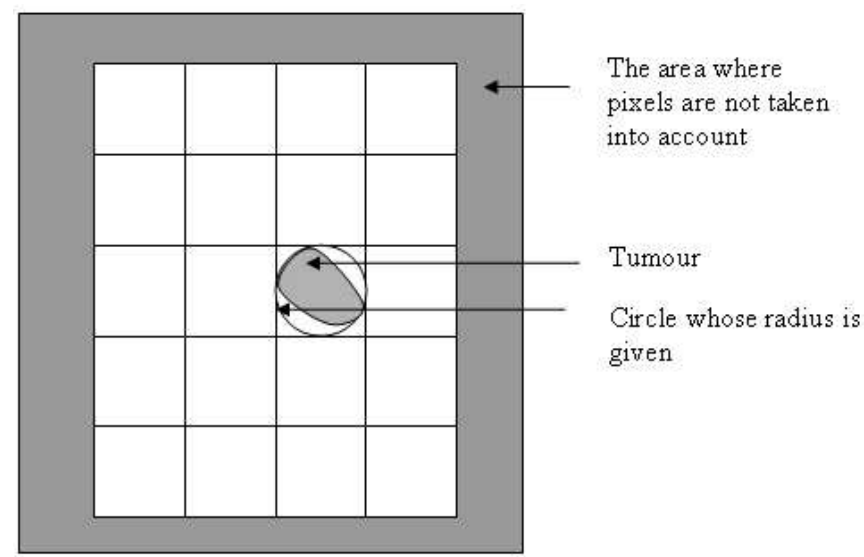

Fig. 1. The possible neighbourhood of the block containing a tumour in a mammogram

found anywhere else in the mammogram. For all the mammograms used in the simulations the information on the location and the size of the tumour is given. From this piece of information, we establish the possible neighbourhood by calculating the number of the blocks with the same size of the block containing the tumour we can have to four corners of the mammogram. In general, for the small tumours, we have a large number of blocks in the possible neighbourhood, whereas for the big tumours, we have small number of blocks in the possible neighbourhood. However, the total number of blocks in the possible neighbourhood also depends on the location of the tumour. All the blocks in the possible neighbourhood are restricted to have equal size, so some pixels near the borders might be neglected. An illustration can be found in Fig. 1. The smallest block in these simulations is $13 \times 13$ and the largest block is $395 \times 395$.

\section{Simulation Results}

From the database [5], the mammograms with tumours are extracted and their identity number remains as shown under (1) in Table 1. For each mammogram, the square block size that contains the tumour can be found under (2) in Table 1. The possible neighbourhood is defined and the number of blocks in the neighbourhood can be found under (3). A set of AR model coefficients is estimated using the method in Section 5. The order of the model is assumed to be $1 \times 1$. Each set of AR model coefficient is compared with the set representing the block with the tumour. The number of sets of AR model coefficients that exceed $\pm 3 \%$ of the coefficients representing the tumour is counted for each mammogram and the results can be found under (4) in Table 1. The percentage of these sets in all blocks in the possible neighbourhood can be obtained under (5) in the same table.

For 100 tumours in 95 mammograms from the database, 38 of them contain no repeating AR model coefficients in their possible neighbourhood. For those 
Table 1. The results arisen from the comparison of two-dimensional autoregressive model coefficients of the tumour block and its possible neighbourhood

\begin{tabular}{|c|c|c|c|c|c|c|c|c|c|}
\hline (1) & (2) & $(3)$ & 4) & (5) & (1) & (2) & $(3)$ & $(4)$ & $(5)$ \\
\hline db001 & $\overline{5 \times 3}$ & 1 & 0 & $\overline{0}$ & $\mathrm{db14}$ & $5 \times 55$ & 323 & 4 & 1.24 \\
\hline & 139 & 41 & 2 & 4.88 & & & $\overline{99}$ & & $\overline{0}$ \\
\hline $55 a$ & $61 \times 61$ & 255 & 1 & .39 & & $49 \times 349$ & 4 & & 0 \\
\hline $5 b$ & $53 \times 53$ & 41 & 2 & 0.59 & & $25 \times 125$ & 48 & & 4.17 \\
\hline mc & $67 \times 67$ & 209 & 1 & .48 & & 99 & 99 & & 2.02 \\
\hline mdl & $81 \times 81$ & 144 & 0 & 0 & 155 & $91 \times 191$ & 19 & & 0 \\
\hline & $63 \times 63$ & 255 & 1 & 0.39 & & $77 \times 177$ & 24 & & 0 \\
\hline $\mathrm{md}$ & $137 \times 137$ & 35 & $\overline{0}$ & 0 & 30 & $123 \times 123$ & 48 & & 2.08 \\
\hline 17 & $97 \times 97$ & 99 & 1 & 1.01 & 63 & $101 \times 101$ & 89 & & $\overline{4.49}$ \\
\hline 19 & $99 \times 99$ & 9 & 2 & 2.02 & 5 & $85 \times 85$ & 120 & & 1.67 \\
\hline 21 & $99 \times 99$ & 80 & 0 & \begin{tabular}{|c|}
0 \\
\end{tabular} & 37 & $71 \times 71$ & 168 & & 0 \\
\hline$m$ & 59 & 271 & 6 & 2.21 & 70 & $165 \times 165$ & 24 & & 0 \\
\hline 25 & $59 \times 159$ & 29 & 0 & & & $25 \times 125$ & 55 & & 9.09 \\
\hline mdl & $113 \times 113$ & 63 & 1 & 1.59 & 175 & $67 \times 67$ & 209 & & 1.9 \\
\hline $\mathbf{m}$ & $87 \times 87$ & 120 & 5 & 4.17 & 178 & $141 \times 141$ & 41 & & 0 \\
\hline 32 & $133 \times 133$ & 48 & 0 & 0 & & $135 \times 135$ & 41 & & 2.8 \\
\hline $\mathrm{md}$ & $55 \times 55$ & 323 & 3 & 0.93 & 181 & $109 \times 109$ & 71 & & $\Omega^{2}$ \\
\hline 63 & 67 & $20 !$ & 9 & 4.31 & 34 & $229 \times 229$ & 16 & & \\
\hline 69 & 89 & 109 & 0 & 0 & & 99 & 100 & & 0 \\
\hline$\overline{72}$ & $57 \times 57$ & 305 & 11 & 3.61 & 188 & $123 \times 123$ & 48 & 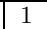 & 2.08 \\
\hline & 47 & 461 & 5 & & & & 23 & & \\
\hline md & 41 & 624 & 3 & 0.48 & & 83 & 120 & & 0 \\
\hline $\mathrm{m}$ & $63 \times 263$ & 8 & $\overline{0}$ & $\bar{c}$ & 93 & $265 \times 265$ & r & & 0 \\
\hline $\mathrm{m}$ & $77 \times 77$ & 155 & 1 & U. & & 53 & 341 & 5 & 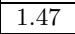 \\
\hline $\mathrm{mc}$ & 99 & 89 & 2 & 2.25 & & $187 \times 187$ & 15 & 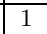 & 6.67 \\
\hline $\mathrm{m}$ & 41 & 599 & 0 & 0 & & 63 & 23 & & 0.84 \\
\hline 92 & 87 & 120 & 2 & 1. & & 75 & 155 & & 1.94 \\
\hline md & $59 \times 59$ & 288 & 10 & 3.47 & 04 & $43 \times 43$ & 528 & 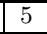 & 0.95 \\
\hline $\mathrm{m}$ & 69 & 195 & 2 & 1. & 206 & 35 & 840 & & 0.71 \\
\hline mc & 47 & 461 & 6 & 1. & 207 & 39 & 675 & 4 & 0.59 \\
\hline 32 & $77 \times 77$ & 143 & 7 & 4.9 & 09 & $175 \times 175$ & 24 & & $\overline{0}$ \\
\hline md & $01 \times 101$ & \begin{tabular}{|l|}
99 \\
\end{tabular} & 1 & 1.0 & 11 & $27 \times 27$ & 1481 & 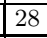 & 1.8 \\
\hline md & $97 \times 197$ & 19 & 0 & 0 & 3 & $1 \times 91$ & 109 & 0 & 0 \\
\hline md & $223 \times 223$ & 15 & 1 & 6.67 & 214 & $23 \times 23$ & $206 !$ & T & 0.04 \\
\hline md & $\overline{03 \times 103}$ & 80 & 3 & 3.75 & 18 & $17 \times 17$ & 3968 & $\overline{2}$ & 0.05 \\
\hline & $235 \times 235$ & 9 & 0 & 0 & & 59 & 271 & 2 & 0.74 \\
\hline md & $69 \times 169$ & 24 & 0 & ( & 22 & 35 & 86 & & 0 \\
\hline md & $59 \times 159$ & 35 & 0 & 0 & $23 a$ & $69 \times 59$ & 271 & $\overline{0}$ & 0 \\
\hline $\mathbf{m c}$ & $165 \times 165$ & 19 & 0 & ( & $23 \mathrm{~b}$ & $13 \times 13$ & 7055 & 18 & 0.255 \\
\hline md & $67 \times 67$ & 209 & 4 & 1.91 & 227 & $19 \times 19$ & 3135 & 63 & 2.01 \\
\hline & $121 \times 121$ & 63 & 0 & 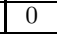 & & $9 \times 89$ & 120 & 3 & 2.5 \\
\hline $\mathrm{mc}$ & $47 \times 47$ & 440 & 8 & 1. & $\overline{236}$ & $29 \times 29$ & 1259 & 3 & 0.24 \\
\hline md & $97 \times 97$ & \begin{tabular}{|l|}
89 \\
\end{tabular} & 1 & 1.12 & 238 & $35 \times 35$ & 840 & 4 & .47 \\
\hline & $57 \times 57$ & 288 & 8 & 2.78 & $239 a$ & $1 \times 81$ & 131 & 0 & 0 \\
\hline $32 a$ & $105 \times 105$ & 72 & 0 & 0 & $239 b$ & $51 \times 51$ & 360 & 23 & 6.39 \\
\hline mdl & $37 \times 37$ & 728 & 6 & 0.82 & 240 & $47 \times 47$ & 440 & 45 & 10.2 \\
\hline mdl & $99 \times 99$ & 89 & 0 & 0 & $\overline{241}$ & $77 \times 77$ & 168 & 2 & 1.19 \\
\hline & $59 \times 59$ & 28 & $\overline{6}$ & 2. & & $105 \times 105$ & & 2 & 3.17 \\
\hline & & 341 & 1 & 0.29 & & $21 \times 21$ & 2499 & 6 & 0.2 \\
\hline db144a & $59 \times 59$ & 88 & 0 & 0 & mdb249 & $97 \times 97$ & 89 & 0 & 0 \\
\hline
\end{tabular}

(1) Mammogram identity number (as in the Database [7]) (2) The size of the block that contains a tumour

(3) The number of blocks available in the entire mammogram.

(4) The number of blocks that can be represented in 2-D AR model coefficients similar to the set representing the tumour block.

(5) The percentage of blocks that contain similar 2-D AR model coefficients compared to the set representing the tumour block. 
who do have a set or sets of AR model coefficients within the range of $\pm 3 \%$ of the AR model coefficients representing the tumour block, mammogram mdb240 contains $10.23 \%$ of the AR model coefficients in all 440 sets estimated which are within this range, followed by mammogram mdb171, which has $9.9 \%$ of its 55 blocks in the neighbourhood within this range of the AR model coefficients representing the tumour. For all the tumours in mammograms we took into account, $98.6 \%$ of the time, the AR model coefficients (within $\pm 3 \%$ ) representing the tumour block does not appear again in any other blocks in the possible neighbourhood.

\section{Conclusion and Summary}

In this work, we looked into the possibility of representing the tumour area of a mammogram using two-dimensional $(2-D)$ autoregressive (AR) models. The possible neighbourhood with blocks of the same size as the one containing a tumour is defined and a set of AR model coefficients is estimated for each block. For all the sets of coefficients obtained, we compared them with the set representing the tumour. It was found from the simulations results that $98.6 \%$ of the time, the AR model coefficients representing the tumour can not be found anywhere else in the same mammogram using the same block size. This lead to the conclusion that $2-D$ AR model coefficients can provide sufficient data for texture analysis of mammograms.

\section{References}

1. S.M. Kay, Modern spectral estimation: theory and application, Prentice Hall 1988.

2. S. Lee and T. Stathaki, "Texture Characterisation Using Constrained Optimisation Techniques with Application to Mammography", Fifth International Workshop on Image Analysis for Multimedia Interactive Services (WIAMIS 2004), on CD-ROM.

3. S. Lee and T. Stathaki, "Two-Dimensional Autoregressive Modelling Using Joint Second and Third Order Statistics and a Weighting Scheme", Twelfth European Signal Processing Conference (EUSIPCO), on CD-ROM, 2004.

4. S. H.-H. Lee, Novel Approaches to Two Dimensional Autoregressive Modelling, Ph.D. and DIC thesis, Imperial College London, 2004.

5. The Mammographic Image Analysis Society (MIAS): MiniMammography Database. http://www.wiau.man.ac.uk/services/MIAS/MIASmini.html, last access: 11th February 2005.

6. T. Stathaki, "2-D autoregrssive modelling using joint and weighted second and third order statistics", Electronics Letters, 32(14) (1996) 1271-1273.

7. J. Suckling, J. Parker, D. Dance, S. Astley, I. Hutt, C. Boggis, I. Ricketts, E. Stamatakis, N. Cerneaz, S. Kok, P. Taylor, D. Betal, and J. Savage, "The Mammographic Image Analysis Society Digital Mammogram Database", Exerpta Medica, International Congress Series, 1069 (1994) 375-378.

8. A. Swami, G.B. Giannakis and J.M. Mendel, "Linear modeling of multidimensional non-Gaussian processes using cumulants", Multidimensional Systems and Signal Processing, 1 (1990) 11-37. 